\title{
Second harmonic generation in partially ordered media and at interfaces: analysis of dynamical and orientational factors
}

\author{
David L. Andrews * , Ian D. Hands \\ School of Chemical Sciences, University of East Anglia, Norwich NR4 7TJ, UK
}

Received 28 March 1996

\begin{abstract}
A new method of modelling molecular orientational dynamics is presented and illustrated by application to optical second harmonic generation (SHG). The method highlights the intricate dependence of the harmonic signal on the form and evolution of the molecular orientational distribution, and reference is made to some examples taken from the recent literature. Specifically, the cases considered relate to SHG in (a) poled polymers, (b) molecules oriented within molecular sieves and (c) molecules in films or adsorbed on surfaces. The technique invokes rotational averages weighted by static or time-dependent distribution functions expressed in terms of Legendre polynomials. Rotational diffusion is used to model the decay of second harmonic intensity associated with a growth or recovery of bulk isotropy, the results allowing several discrete contributions associated with different time constants to be characterised. For polarisation studies using surfaces and films, it is shown that caution is required in how the results are interpreted to reflect molecular orientation, approximations in commonly employed theory readily producing spurious results.
\end{abstract}

\section{Introduction}

It is well known that coherent second harmonic generation (SHG) cannot occur in single-phase molecular fluids possessing macroscopic isotropy [1]. In situations where such isotropy can be removed or is naturally lacking, SHG is allowed and can hence provide a very sensitive tool for detecting preferential molecular orientation. For example SHG has been used to gauge molecular orientation: at fluid interfaces [2,3]; within cell membranes [4,5]; of dipolar chromophores in poled polymers [6,7] and of molecules within molecular sieves [8]. In circumstances where preferential orientation arises, theory requires the application of suitably weighted three-dimensional rotational ensemble averages in order to arrive at the nett media response.

The weighting of orientational averages by phase factors of the form $\exp (i \hat{\boldsymbol{u}} \cdot \hat{\boldsymbol{w}})$, where $\hat{\boldsymbol{u}}$ and $\hat{\boldsymbol{w}}$ are laboratory and molecule-fixed vectors respectively, is important for consideration of pairwise interactions of molecules with radiation, and has been treated in some detail [9]. As indicated in Ref. [9] the form of the derivation is easily extended to cover averages weighted by a Boltzmann factor and may be used, for example, to derive an expression for steady state electric field induced second harmonic generation (EFISHG). In this

\footnotetext{
${ }^{*}$ Corresponding author. Fax: +44603259855.
} 
paper we consider how use may be made, under appropriate conditions, of rotational averages weighted by Legendre polynomials. The specific application to SHG is considered, and detailed comparisons are made with various experimental results in the recent literature.

\section{Weighted rotational averages}

Let $\hat{\boldsymbol{w}}$ define a unit reference vector fixed within the molecule of interest and $\hat{\boldsymbol{u}}$ be a unit laboratory-fixed reference vector. We shall consider the case of an ensemble of molecules such that the distribution of molecular orientations may be specified at any time $t$ by the function $f(\theta, t)$ where $\theta$ is the angle between the two reference directions $\hat{\boldsymbol{u}}$ and $\hat{\boldsymbol{w}}$ so that $f(\theta, t) \mathrm{d} \theta$ is the fractional number of molecules with their $\hat{w}$ vector set at an angle between $\theta$ and $\theta+\mathrm{d} \theta$ with respect to the laboratory fixed vector $\hat{\boldsymbol{u}}$. A time dependence is included to accommodate the situation in which preferentially oriented molecules suddenly become free of their orientating influence and undergo reorientation back to an isotropic distribution. We shall assume that the relaxation occurs via rotational diffusion and may therefore be described by [10]

$$
\frac{\xi}{k T} \frac{\partial f}{\partial t}=\frac{1}{\sin \theta} \frac{\partial}{\partial \theta}\left(\sin \theta \frac{\partial f}{\partial \theta}\right)
$$

where $k$ is Boltzmann's constant, $T$ is the absolute temperature and $\xi$ is a quantity referred to as the "inner friction"' by Debye. Making the substitution

$$
c=\cos \theta
$$

this equation may be transformed to

$$
\frac{\xi}{k T} \frac{\partial f}{\partial t}=\frac{\partial}{\partial c}\left(\left(1-c^{2}\right) \frac{\partial f}{\partial c}\right)
$$

The parameter $c$ is restricted to the interval $[-1,1]$ over which the Legendre polynomials form a complete basis set, and so we can expand $f$ in the form

$$
f(c, t)=\sum_{n=0}^{\infty} a_{n}(t) P_{n}(c),
$$

where $P_{n}(c)$ is the Legendre polynomial of order $n$. This expansion is quite general and should be applicable to any distribution where the probability of a certain molecular orientation depends only on the angle between that orientation and a fixed laboratory direction. Substituting (4) into (3) and using the defining relations for the Legendre polynomials,

$$
\left(1-c^{2}\right) \frac{d^{2} P_{n}(c)}{\mathrm{d} c^{2}}-2 c \frac{\mathrm{d} P_{n}(c)}{\mathrm{d} c}+n(n+1) P_{n}(c)=0,
$$

we obtain the differential equation for the coefficients $a_{n}(t)$;

$$
\frac{\mathrm{d} a_{n}(t)}{\mathrm{d} t}=-\frac{n(n+1) k T}{\xi} a_{n}(t),
$$

with the solution

$$
a_{n}(t)=a_{n}(0) \exp \left(-\frac{n(n+1) k T}{\xi} t\right)=a_{n}(0) \exp \left(-\frac{n(n+1)}{2 \tau} t\right),
$$


Table 1

Legendre polynomial weighted rotational averages of direction cosines products; $n$ is the rank of the tensor to be averaged and $m$ the order of the weighting Legendre polynomial

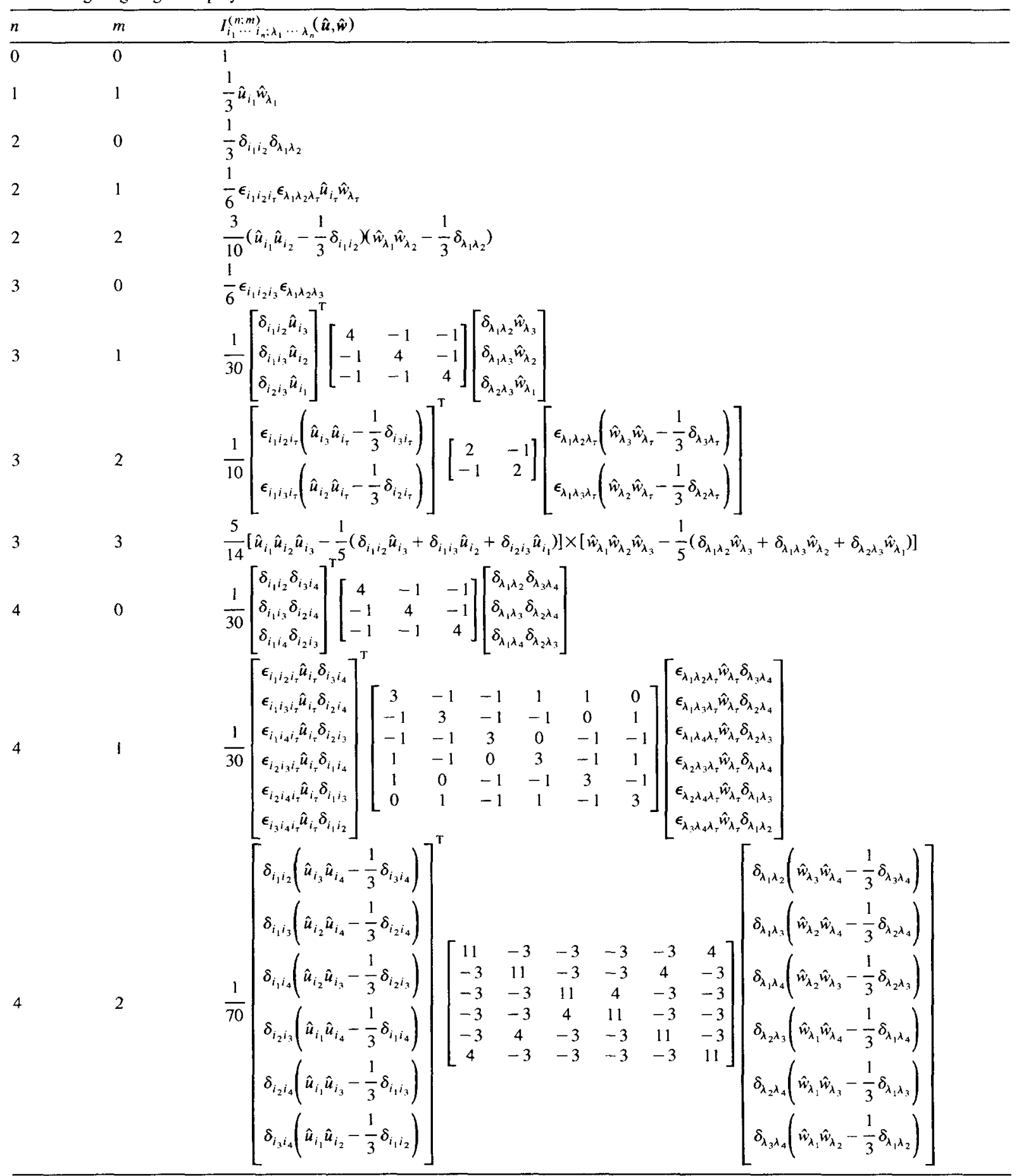


Table 1 (continued)

$$
\begin{aligned}
& \text { n } \quad m \quad I_{i_{1} ; \cdots i_{n}: \lambda_{1} \cdots \lambda_{n}}^{(n ; m)}(\hat{u}, \hat{w}) \\
& 43 \frac{5}{56}\left[\begin{array}{c}
\epsilon_{i_{1} i_{3} i_{\tau}}\left[\hat{u}_{i_{2}} \hat{u}_{i_{4}} \hat{u}_{i_{\tau}}-\frac{1}{5}\left(\delta_{i_{2} i_{4}} \hat{u}_{i_{\tau}}+\delta_{i_{4} i_{\tau}} \hat{u}_{i_{2}}+\delta_{i_{2} i_{4}} \hat{u}_{i_{\tau}}\right)\right] \\
\epsilon_{i_{2} i_{3} i_{\tau}}\left[\hat{u}_{i_{1}} \hat{u}_{i_{4}} \hat{u}_{i_{\tau}}-\frac{1}{5}\left(\delta_{i_{1} i_{\tau}} \hat{u}_{i_{4}}+\delta_{i_{4} i_{\tau}} \hat{u}_{i_{1}}+\delta_{i_{1} i_{4}} \hat{u}_{i_{\tau}}\right)\right] \\
\epsilon_{i_{3} i_{4} i_{\tau}}\left[\hat{u}_{i_{1}} \hat{u}_{i_{2}} \hat{u}_{i_{\tau}}-\frac{1}{5}\left(\delta_{i_{1} i_{\tau}} \hat{u}_{i_{2}}+\delta_{i_{2} i_{\tau}} \hat{u}_{i_{1}}+\delta_{i_{1} i_{2}} \hat{u}_{i_{\tau}}\right)\right]
\end{array}\right]^{\mathrm{T}}
\end{aligned}
$$

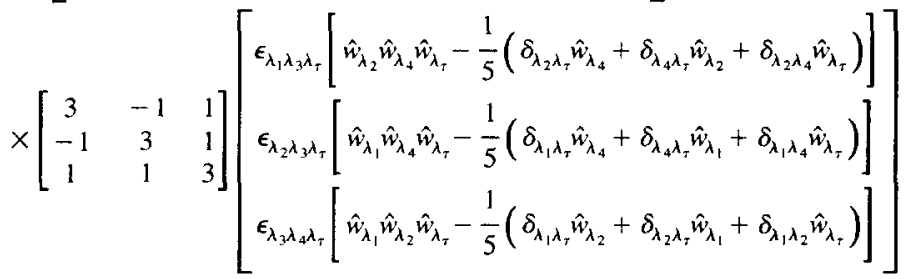

$$
\begin{aligned}
& 44 \frac{35}{72}\left[\hat{u}_{i_{1}} \hat{u}_{i_{2}} \hat{u}_{i_{3}} \hat{u}_{i_{4}}-\frac{1}{7}\left(\delta_{i_{1} i_{2}} \hat{u}_{i_{3}} \hat{u}_{i_{4}}+\delta_{i_{1} i_{3}} \hat{u}_{i_{2}} \hat{u}_{i_{4}}+\delta_{i_{1} i_{4}} \hat{u}_{i_{2}} \hat{u}_{i_{3}}+\delta_{i_{2} i_{3}} \hat{u}_{i_{1}} \hat{u}_{i_{4}}+\delta_{i_{2} i_{4}} \hat{u}_{i_{1}} \hat{u}_{i_{3}}+\delta_{i_{3} i_{4}} \hat{u}_{i_{1}} \hat{u}_{i_{2}}\right)\right. \\
& \left.+\frac{1}{35}\left(\delta_{i_{1} i_{2}} \delta_{i_{3} i_{4}}+\delta_{i_{1} i_{3}} \delta_{i_{2} i_{4}}+\delta_{i_{1} i_{4}} \delta_{i_{2} i_{3}}\right)\right]\left[\hat{w}_{\lambda_{1}} \hat{w}_{\lambda_{2}} \hat{w}_{\lambda_{3}} \hat{w}_{\lambda_{4}}-\frac{1}{7}\left(\delta_{\lambda_{1} \lambda_{2}} \hat{w}_{\lambda_{3}} \hat{w}_{\lambda_{4}}+\delta_{\lambda_{1} \lambda_{3}} \hat{w}_{\lambda_{2}} \hat{w}_{\lambda_{4}}+\delta_{\lambda_{1} \lambda_{4}} \hat{w}_{\lambda_{2}} \hat{w}_{\lambda_{3}}+\delta_{\lambda_{2} \lambda_{3}} \hat{w}_{\lambda_{1}} \hat{w}_{\lambda_{4}}+\delta_{\lambda_{2} \lambda_{4}} \hat{w}_{\lambda_{1}} \hat{w}_{\lambda_{3}}\right.\right. \\
& \left.\left.+\delta_{i_{3} j_{4}} \hat{w}_{\lambda_{1}} \hat{w}_{\lambda_{2}}\right)+\frac{1}{35}\left(\delta_{\lambda_{1} \lambda_{2}} \delta_{\lambda_{3} \lambda_{4}}+\delta_{\lambda_{1} \lambda_{3}} \delta_{\lambda_{2} \lambda_{4}}+\delta_{\lambda_{1} \lambda_{4}} \delta_{\lambda_{2} \lambda_{3}}\right)\right]
\end{aligned}
$$

introducing a characteristic relaxation time $\tau$ defined as $\tau=\xi / 2 k T$. The coefficients thus "relax" at different rates except for $a_{0}$ which is time independent. The expression for $f$ now becomes

$$
f(c, t)=\sum_{n=0}^{\infty} a_{n}(0) \exp \left(-\frac{n(n+1)}{2 \tau} t\right) P_{n}(c) \text {. }
$$

Let $f_{0}$ represent the distribution function at $t=0$ so that

$$
f_{0}=f(c, 0)=\sum_{n=0}^{\infty} a_{n}(0) P_{n}(c) \text { : }
$$

by using the orthogonality relations

$$
\int_{-1}^{1} P_{m}(c) P_{n}(c) \mathrm{d} c=\frac{2}{2 m+1} \delta_{m n}
$$

we can deduce that

$$
a_{n}(0)=\frac{2 n+1}{2} \int_{-1}^{1} f_{0} P_{n}(c) \mathrm{d} c .
$$

We are particularly interested in averaging properties $S$ that are generally expressible in the form

$$
S=T_{i_{1}} \ldots i_{n} V_{i_{1}} \cdots i_{n},
$$

where $T_{i} \ldots i_{n}$ is a rank- $n$ tensor representing the external conditions applied to a typical molecule of the fluid ensemble, $V_{i_{1}}^{n} \ldots i_{n}$ is a rank- $n$ tensor representing the molecular response, and repeated tensor indices imply summation over a three-dimensional Cartesian set. The property $S$, therefore, is generally dependent on 
molecular orientation. Due to the chosen form of $f$, rotational averaging will therefore involve the evaluations of

$$
\left\langle T_{i_{1} \cdots i_{n}} V_{i_{1} \cdots i_{n}} P_{m}(\hat{\boldsymbol{u}} \cdot \hat{\boldsymbol{w}})\right\rangle_{\Omega}=T_{i_{1} \cdots i_{n}} V_{\lambda_{1} \cdots \lambda_{n}} I_{i_{1} \cdots i_{n} ; \lambda_{1} \cdots \lambda_{n}}^{(n ; m)}(\hat{\boldsymbol{u}}, \hat{\boldsymbol{w}}),
$$

where, by an obvious adaptation of the standard terminology, the weighted average contributions $I^{(n ; m)}$ are defined by

$$
I_{i_{1} \cdots i_{n} ; \lambda_{1} \cdots \lambda_{n}}^{(n ; m)}(\hat{\boldsymbol{u}}, \hat{\boldsymbol{w}})=\left\langle l_{i_{1} \lambda_{1}} \cdots l_{i_{n} \lambda_{n}} P_{m}(\hat{\boldsymbol{u}} \cdot \hat{\boldsymbol{w}})\right\rangle_{\Omega},
$$

$l_{i_{r} \lambda_{r}}$ being the direction cosine between the $i_{r}$ component of the laboratory fixed axes and the $\lambda_{r}$ component of the molecule fixed axes. In Eqs. (13) and (14) the subscript $\Omega$ is used to denote an orientational average; a distributional average taken over the positions of the molecular centres will be denoted by a subscript $R$. The form of these polynomial weighted averages may be gleaned from Ref. [9] and are given in Table 1 for tensors up to rank 4 . These averages may now be used to evaluate the necessary ensemble averages, the process being illustrated by considering SHG from oriented molecules.

\section{Second harmonic generation from oriented molecules}

The general expression for the intensity $I_{2 \omega}$ of optical second harmonic generation from a collection of $N$ molecules may be derived using quantum electrodynamics [1]:

$$
I_{2 \omega}=\mathscr{D}^{(2)}\left|\sum_{\xi}^{N} \beta_{i j k}^{(\xi)} \bar{e}_{i}^{\prime} e_{j} e_{k} \exp \left(\mathrm{i} \Delta \boldsymbol{k} \cdot \boldsymbol{R}_{\xi}\right)\right|^{2},
$$

where $\Delta \boldsymbol{k}=2 \boldsymbol{k}-\boldsymbol{k}^{\prime}$ is the wave vector mismatch between the fundamental input beam (wave vector $\boldsymbol{k}$, frequency $\omega=c|k|$, irradiance $I_{\omega}$ ) and the emergent second harmonic beam (wave vector $\boldsymbol{k}^{\prime}$, frequency $\omega^{\prime}=2 \omega$, irradiance $I_{2 \omega}$ ). In Eq. (15), $R_{\xi}$ is the position vector of the molecule $\xi, \beta^{(\xi)}$ is the associated molecular hyperpolarisability symmetrized in its last two indices, $\boldsymbol{e}$ and $\boldsymbol{e}^{\prime}$ are the polarisation vectors of the fundamental and harmonic beams respectively (an overbar being used to represent the complex conjugate); finally, $\mathscr{D}^{(2)}$ is a beam parameter given by

$$
\mathscr{D}^{(2)} \approx \frac{k^{4} I_{\omega}^{2} g_{\omega}^{(2)}}{32 \pi^{2} c \epsilon_{0}^{3}}
$$

with $g_{\omega}^{(2)}$ the input degree of second order coherence.

Eq. (15) can be written in the form

$$
I_{2 \omega}=\mathscr{D}^{(2)}\left(\sum_{\xi}^{N} \sum_{\xi^{\prime}}^{N} \beta_{i j k}^{(\xi)} \bar{e}_{i}^{\prime} e_{j} e_{k} \bar{\beta}_{l m n}^{\left(\xi^{\prime}\right)} e_{i}^{\prime} \bar{e}_{m} \bar{e}_{n} \exp \left(\mathrm{i} \Delta \boldsymbol{k} \cdot \boldsymbol{R}_{\xi \xi^{\prime}}\right)\right),
$$

where $\boldsymbol{R}_{\xi \xi^{\prime}}=\boldsymbol{R}_{\xi}-\boldsymbol{R}_{\xi^{\prime}}$. The expression (17) can now be written as the sum of two terms,

$$
I_{2 \omega}=I_{2 \omega}^{\mathrm{inc}}+I_{2 \omega}^{\mathrm{coh}},
$$

with

$$
l_{2 \omega}^{\text {inc }}=\mathscr{D}^{(2)}\left(\sum_{\xi}^{N} \beta_{i j k}^{(\xi)} \bar{e}_{i}^{\prime} e_{j} e_{k} \bar{\beta}_{l m n}^{(\xi)} e_{l}^{\prime} \bar{e}_{m} \bar{e}_{n}\right)
$$


representing the incoherent SHG from individual molecules and

$$
I_{2 \omega}^{\mathrm{coh}}=\mathscr{D}^{(2)}\left(\sum_{\xi \neq \xi^{\prime}}^{N} \sum_{\xi^{\prime}}^{N} \beta_{i j k}^{(\xi)} \bar{e}_{i}^{\prime} e_{j} e_{k} \bar{\beta}_{l m n}^{\left(\xi^{\prime}\right)} e_{l}^{\prime} \bar{e}_{m} \bar{e}_{n} \exp \left(\mathrm{i} \Delta \boldsymbol{k} \cdot \boldsymbol{R}_{\xi \xi^{\prime}}\right)\right)
$$

the coherent SHG associated with interference between photons generated at different molecular centres. Assuming that the variables describing the orientation of different molecules are independent of one another and that the molecules are identical, the ensemble average of (19) becomes

$$
\left\langle I_{2 \omega}^{\mathrm{inc}}\right\rangle=\mathscr{D}^{(2)}\left(\sum_{\xi}^{N}\left\langle\beta_{i j k}^{(\xi)} \bar{e}_{i}^{\prime} e_{j} e_{k} \bar{\beta}_{l m n}^{(\xi)} e_{l}^{\prime} \bar{e}_{m} \bar{e}_{n}\right\rangle_{\Omega}\right)=\mathscr{D}^{(2)} N\left\langle\left|\beta_{i j k} \bar{e}_{i}^{\prime} e_{j} e_{k}\right|^{2}\right\rangle_{\Omega},
$$

the angular brackets now representing an average over the orientational coordinates of a single molecule. Taking the ensemble average of the coherent SHG signal, and assuming the spatial and orientational degrees of freedom are also separable, we have

$$
\begin{aligned}
\left\langle I_{2 \omega}^{\mathrm{coh}}\right\rangle & =\mathscr{D}^{(2)}\left(\sum_{\xi \neq \xi^{\prime}}^{N} \sum_{\xi^{\prime}}^{N}\left\langle\boldsymbol{\beta}_{i j k}^{(\xi)} \bar{e}_{i}^{\prime} e_{j} e_{k}\right\rangle_{\Omega}\left\langle\overline{\boldsymbol{\beta}}_{l m n}^{\left(\xi^{\prime}\right)} e_{l}^{\prime} \bar{e}_{m} \bar{e}_{n}\right\rangle_{\Omega}\left\langle\exp \left(\mathrm{i} \Delta \boldsymbol{k} \cdot \boldsymbol{R}_{\xi \xi^{\prime}}\right)\right\rangle_{\mathrm{R}}\right) \\
& =\left.\mathscr{D}^{(2)}\left\langle\boldsymbol{\beta}_{i j k} \bar{e}_{i}^{\prime} e_{j} e_{k}\right\rangle_{\Omega}\right|^{2} \sum_{\xi \neq \xi^{\prime}}^{N} \sum_{\xi^{\prime}}^{N}\left\langle\exp \left(\mathrm{i} \Delta \boldsymbol{k} \cdot \boldsymbol{R}_{\xi \xi^{\prime}}\right)\right\rangle_{\mathrm{R}} .
\end{aligned}
$$

To proceed further, we first define the following beam parameter, which fully accommodates features of the beam input and the experimental geometry;

$$
\mathscr{B}^{(2)}(\Delta \boldsymbol{k})=\mathscr{D}^{(2)} \sum_{\xi \neq \xi^{\prime}}^{N} \sum_{\xi^{\prime}}^{N}\left\langle\exp \left(\mathrm{i} \Delta \boldsymbol{k} \cdot \boldsymbol{R}_{\xi \xi^{\prime}}\right)\right\rangle_{\mathrm{R}},
$$

such that the expression for the coherent second harmonic signal $\mathscr{I}$ is

$$
\mathscr{I}=\left\langle I_{2 \omega}^{\mathrm{cob}}\right\rangle=\mathscr{B}^{(2)}(\Delta \boldsymbol{k})\left|\left\langle\beta_{i j k} \bar{e}_{i}^{\prime} e_{j} e_{k}\right\rangle_{\Omega}\right|^{2}
$$

The dependence of $\mathscr{B}^{(2)}(\Delta \boldsymbol{k})$ on the wave vector mismatch is written as a reminder that Eq. (24) will correctly account for the polarisation variation of the intensity of the second harmonic signal for a fixed wave vector mismatch. The weighted average in Eq. (24) is given by

$$
\left\langle\beta_{i j k} \bar{e}_{i}^{\prime} e_{j} e_{k}\right\rangle_{\Omega}=\left\langle\beta_{i j k} \bar{e}_{i}^{\prime} e_{j} e_{k} f(c, t)\right\rangle_{\Omega} /\langle f(c, t)\rangle_{\Omega}=\frac{\sum_{n=0}^{\infty} a_{n}(t)\left\langle\beta_{i j k} \bar{e}_{i}^{\prime} e_{j} e_{k} P_{n}(c)\right\rangle_{\Omega}}{\sum_{n=0}^{\infty} a_{n}(t)\left\langle P_{n}(c)\right\rangle_{\Omega}},
$$

where Eq. (4) has been used for the weighting function $f$. Using Eq. (13) and Table 1 the expression for the second harmonic intensity becomes

$$
\mathscr{I}=\mathscr{B}^{(2)}(\Delta \boldsymbol{k})\left|\frac{\beta_{\lambda \mu \nu} \bar{e}_{i}^{\prime} e_{j} e_{k}}{a_{0}} \sum_{n=0}^{3} a_{n}(t) I_{i j k ; \lambda \mu \nu}^{(3 ; n)}(\hat{\boldsymbol{u}}, \hat{\boldsymbol{w}})\right|^{2} .
$$

In the case of relaxation due to rotational diffusion this may be written

$$
\mathscr{I}=\mathscr{B}^{(2)}(\Delta \boldsymbol{k})\left|\frac{1}{a_{0}} \sum_{n=0}^{3} a_{n}(0) B_{n}(\hat{\boldsymbol{u}}, \hat{\boldsymbol{w}}) \exp \left(-\frac{n(n+1)}{2 \tau} t\right)\right|^{2}
$$


Table 2

Explicit form of the terms $B_{n}(\hat{u}, \hat{w})$ contributing to second harmonic generation as given by Eq. (27), revealing their polarisation dependence

\begin{tabular}{ll}
\hline$n$ & $B_{n}(\hat{u}, \hat{w})$ \\
\hline 0 & 0 \\
1 & $\frac{1}{15}\left[\left(2 \beta_{\lambda \mu \mu} \hat{w}_{\lambda}-\beta_{\lambda \lambda \mu} \hat{w}_{\mu}\right)(\boldsymbol{e} \cdot \boldsymbol{e})\left(\overline{\boldsymbol{e}}^{\prime} \cdot \hat{\boldsymbol{u}}\right)+\left(3 \beta_{\lambda \lambda \mu} \hat{w}_{\mu}-\beta_{\lambda \mu \mu} \hat{w}_{\lambda}\right)\left(\overrightarrow{\boldsymbol{e}}^{\prime} \cdot \boldsymbol{e}\right)(\boldsymbol{e} \cdot \hat{\boldsymbol{u}})\right]$ \\
2 & $\frac{1}{5} \epsilon_{\lambda \mu \tau} \beta_{\lambda \mu \nu} \hat{w}_{\nu} \hat{w}_{\tau}(\boldsymbol{e} \cdot \hat{\boldsymbol{u}})\left(\overline{\boldsymbol{e}}^{\prime} \times \boldsymbol{e}\right) \cdot \hat{\boldsymbol{u}}$ \\
3 & $\frac{1}{70}\left(5 \beta_{\lambda \mu \nu} \hat{w}_{\lambda} \hat{w}_{\mu} \hat{w}_{\nu}-2 \beta_{\lambda \lambda \mu} \hat{w}_{\mu}-\beta_{\lambda \mu \mu} \hat{w}_{\lambda}\right)\left[5\left(\overline{\boldsymbol{e}}^{\prime} \cdot \hat{\boldsymbol{u}}\right)(\boldsymbol{e} \cdot \hat{\boldsymbol{u}})^{2}-2\left(\overline{\boldsymbol{e}}^{\prime} \cdot \boldsymbol{e}\right)(\boldsymbol{e} \cdot \hat{\boldsymbol{u}})-(\boldsymbol{e} \cdot \boldsymbol{e})\left(\overline{\boldsymbol{e}}^{\prime} \cdot \hat{\boldsymbol{u}}\right)\right]$ \\
\hline
\end{tabular}

where

$$
B_{n}(\hat{\boldsymbol{u}}, \hat{\boldsymbol{w}})=I_{i j k ; \lambda \mu \nu}^{(3: n)}(\hat{\boldsymbol{u}}, \hat{\boldsymbol{w}}) \beta_{\lambda \mu \nu} \bar{e}_{i}^{\prime} e_{j} e_{k} .
$$

Table 1 may now be used to evaluate these coefficients - the results are shown in Table 2. Note immediately that the $n=0$ term disappears; the sum in Eq. (27) therefore contains, at most, three exponential decay terms relaxing at rates in the ratio $1: 3: 6$. This corresponds to a second harmonic signal in whose relaxation it is possible to identify (at most) six contributions with rate constants in the ratio $2: 4: 6: 7: 9: 12$. The number of distinct relaxation rates would be smaller if any of the coefficients $a_{n}$ or $B_{n}(\hat{\boldsymbol{u}}, \hat{\boldsymbol{w}})$ became equal to zero. The representation of experimentally measured data in terms of multiple exponential form is thus given a sound theoretical basis. Note also that all the $B_{n}(\hat{\boldsymbol{u}}, \hat{\boldsymbol{w}})$ of Table 2 vanish identically if $\hat{\boldsymbol{u}}, \boldsymbol{k}$ and $\boldsymbol{k}^{\prime}$ are collinear, as follows from $(\boldsymbol{k} \cdot \boldsymbol{e})=\left(\boldsymbol{k}^{\prime} \cdot \boldsymbol{e}^{\prime}\right)=0$. Thus, no coherent second harmonic will emerge from a molecular system whose direction of preferred orientation is aligned with the radiation throughput.

To illustrate the above theory we now consider three examples taken from the literature and show, in particular, how the theory can be used to derive the dependence of SHG on the polarisation of the second harmonic and fundamental beams.

\section{Applications}

\subsection{Relaxation after alignment in an electrostatic field}

On application of an electrostatic field $E$ and subsequent attainment of equilibrium conditions, the orientational distribution may be found by solving the time independent version of Eq. (3) $(\partial f / \partial t=0)$. The result obtained is

$$
f_{0}=\frac{x \mathrm{e}^{x c}}{2 \sinh x},
$$

where, $x=\mu E_{\mathrm{loc}} / k T, \mu$ being the magnitude of the permanent molecular dipole moment, $E_{\mathrm{loc}}$ is the local static field strength expressible in terms of the applied field through the usual Lorentz factor and $c$ now the cosine of the angle between the electrostatic field and dipole moment vectors. Use of Eqs. (11) and (27) now provides an expression for the second harmonic signal from the relaxing molecules, assuming that the molecules relax via rotational diffusion:

$$
\begin{aligned}
\mathscr{J}= & \mathscr{B}^{(2)}(\Delta \boldsymbol{k}) \mid 3(\operatorname{coth} x-1 / x) B_{1}(\hat{\boldsymbol{E}}, \hat{\boldsymbol{\mu}}) \mathrm{e}^{-t / \tau}+5\left(1-3 \operatorname{coth} x / x-3 / x^{2}\right) B_{2}(\hat{\boldsymbol{E}}, \hat{\boldsymbol{\mu}}) \mathrm{e}^{-3 t / \tau} \\
& +\left.7\left(\operatorname{coth} x-6 / x+15 \operatorname{coth} x / x^{2}-15 / x^{3}\right) B_{3}(\hat{\boldsymbol{E}}, \hat{\boldsymbol{\mu}}) \mathrm{e}^{-6 / \tau}\right|^{2}
\end{aligned}
$$




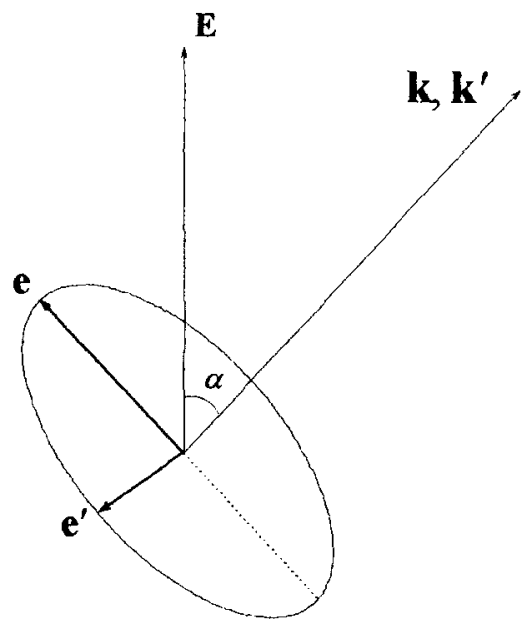

Fig. 1. Experimental arrangement that could be utilised to observe the SHG arising from the $B_{2}(\hat{\boldsymbol{E}}, \hat{\boldsymbol{\mu}})$ term in Eq. (31).

In most molecular systems the strength of the applied electric field and the magnitude of the molecular dipole moment are such that $x \ll 1$. For such cases, Eq. (30) reduces to

$$
\begin{aligned}
\mathscr{I}= & \mathscr{B}^{(2)}(\Delta k) \mid x B_{1}(\hat{\boldsymbol{E}}, \hat{\boldsymbol{\mu}}) \mathrm{e}^{-t / \tau}+\left(x^{2} / 3\right) B_{2}(\hat{\boldsymbol{E}}, \hat{\boldsymbol{\mu}}) \mathrm{e}^{-3 t / \tau} \\
& -\left.\left(x^{3} / 15\right)\left[B_{1}(\hat{\boldsymbol{E}}, \hat{\boldsymbol{\mu}}) \mathrm{e}^{-t / \tau}-B_{3}(\hat{\boldsymbol{E}}, \hat{\boldsymbol{\mu}}) \mathrm{e}^{-6 t / \tau}\right]\right|^{2},
\end{aligned}
$$

where expansion terms involving the fourth and higher powers of $x$ are safely ignored. Generally the field is applied perpendicularly to the beam propagation direction to maximise the output, as follows from detailed consideration of the $B_{n}(\hat{\boldsymbol{u}}, \hat{\boldsymbol{w}})$ given by Table 2 (see also Ref. [14]).

The leading term in Eq. (31) leads us to expect a second harmonic signal that decays exponentially, and with a pre-exponential factor that is inversely proportional to the square of the absolute temperature. However, it is not difficult to arrange an experiment in which the first term cannot contribute to the SHG. For example, consider the arrangement shown in Fig. 1. Here the fundamental propagates at a (non-zero) angle $\alpha$ to the direction $\hat{\boldsymbol{a}}=\hat{\boldsymbol{E}}$ in which the static field was previously applied and is plane polarised in the $(\hat{E}, \hat{\boldsymbol{k}})$ plane formed by the static field and propagation directions. The second harmonic signal is observed in the forward direction such that only photons polarised perpendicularly to the aforementioned plane are detected. Evaluating the expressions for the $B_{n}(\hat{\boldsymbol{u}}, \hat{\boldsymbol{w}})$ terms in Table 2 shows that in this configuration both $B_{1}(\hat{\boldsymbol{E}}, \hat{\boldsymbol{\mu}})$ and $B_{3}(\hat{\boldsymbol{E}}, \hat{\boldsymbol{\mu}})$ disappear and we are left with

$$
\begin{aligned}
\mathscr{I} & =\mathscr{B}^{(2)}(\Delta k)\left|\frac{1}{2}\left(1-3 \operatorname{coth} x / x-3 / x^{2}\right) \sin 2 \alpha \epsilon_{\lambda \mu \tau} \beta_{\lambda \mu \nu} \hat{\mu}_{\nu} \hat{\mu}_{\tau} \mathrm{e}^{-3 t / \tau}\right|^{2} \\
& \approx \mathscr{B}^{(2)}(\Delta k)\left|\left(x^{2} / 30\right) \sin 2 \alpha \epsilon_{\lambda \mu \tau} \beta_{\lambda \mu \nu} \hat{\mu}_{\nu} \hat{\mu}_{\tau} \mathrm{e}^{-3 t / \tau}\right|^{2} .
\end{aligned}
$$

Here the SHG decays exponentially with a rate constant three times larger than in the more usual beam geometry, ( $\boldsymbol{E}$ perpendicular to $\boldsymbol{k}$ ), as indicated by Eq. (31) and we also expect to find a pre-exponential factor whose temperature dependence now varies as $T^{-4}$.

One possible application of this theory lies in polymer dynamics. Polymers containing dipolar chromophores may be "poled" at elevated temperatures by the application of a strong electrostatic field. Cooling the polymer in the presence of the field traps the chromophores so that the polymer retains its nonlinear optical character. It is thought that polymers treated in this way could serve as useful materials for construction of electrooptic devices; the large hyperpolarisabilities of well chosen organic molecules and formation of components of 


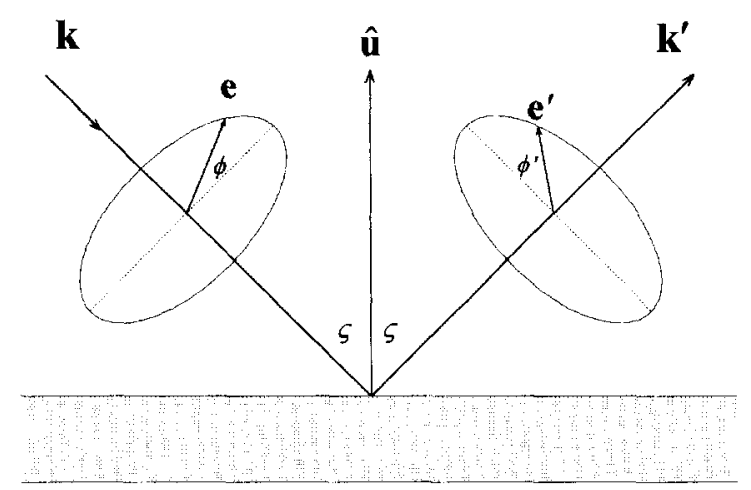

Fig. 2. Defining geometry for the reflection SHG expressions. The reference direction $\hat{\boldsymbol{u}}$ (coincident with $\boldsymbol{E}$ for poled polymers) is taken to be normal to the surface and $s$ is the angle of incidence (reflection) of the fundamental (second harmonic) beam. The angles $\phi$, $\phi^{\prime}$ are positive in the sense of an anti-clockwise rotation from the $\left(\boldsymbol{k}, \boldsymbol{k}^{\prime}\right)$ plane about the positive $k, \boldsymbol{k}^{\prime}$ directions.

complex shape being just some likely advantages [11]. However, the stability of a poled polymer is a major concern, the "freezing in" technique suffering from random diffusional processes which degrade the SHG efficiency over a period of time. Methods which permanently link the oriented chromophores via chemical bonds are obviously desirable in this respect. Clearly the representation of the signal's time evolution discussed in Section 2 will not apply to such poled polymers - free rotational diffusion hardly being an appropriate model for solid polymers in which chromophores are entangled and trapped. However, over a period of time short enough for orientational stability to be assumed, the chromophore distribution will be effectively static and, whatever its form, amenable to expansion in terms of Legendre polynomials. Thus it is appropriate to use Eq. (3), with $\partial f / \partial t=0$, provided the chromophore distribution only depends on the angle $\theta$, the angle between the chromophore molecular dipole moment and the poling direction. Following the analysis through with a time independent distribution therefore shows that the SHG from the poled polymer should have the polarisation dependence indicated by Eq. (27) with $t=0$. Polarisation studies on such a polymer should therefore enable one to confirm or refute the validity of the assumption of a distribution dependent only on $\theta$.

At higher temperatures, for example at the temperature at which such a polymer is poled, some correspondence might be expected between the theory of temporal development given here and the experimentally observed relaxation. By studying the polarisation of second harmonic emissions during poling-relaxation cycles at the poling temperature, information may be available regarding the relaxation processes and prove useful in developing poled polymers of greater stability. One particular study [7] made at temperatures near the glass transition temperature of poled N-(4-nitrophenyl)-(S)-prolinoxypoly(p-hydroxystyrene) films is interesting because it reports SHG data for a variety of poling conditions. Adopting the surface geometry shown in Fig. 2 , defining $s$ as the angle of incidence and with $\phi, \phi^{\prime}$ as shown, the expression for the second harmonic intensity from Eq. (30) is

$$
\begin{aligned}
\mathscr{I}= & \frac{\mathscr{B}^{(2)}(\Delta k)}{1600} \mid 8(\operatorname{coth} x-1 / x) \sin \varsigma\left[\left(2 \beta_{\lambda \mu \mu} \hat{\mu}_{\lambda}-\beta_{\lambda \lambda \mu} \hat{\mu}_{\mu}\right) \cos \phi^{\prime}\right. \\
& \left.+\left(3 \beta_{\lambda \lambda \mu} \hat{\mu}_{\mu}-\beta_{\lambda \mu \mu} \hat{\mu}_{\lambda}\right) \cos \phi\left(\sin \phi \sin \phi^{\prime}-\cos \phi \cos \phi^{\prime} \cos 2 \varsigma\right)\right] \mathrm{e}^{-t / \tau} \\
& -20\left(1-3 \operatorname{coth} x / x-3 / x^{2}\right)\left[\epsilon_{\lambda \mu \tau} \beta_{\lambda \mu \nu} \hat{\mu}_{\nu} \mu_{\tau} \cos \phi \sin \left(\phi+\phi^{\prime}\right) \sin 2 \varsigma\right] \mathrm{e}^{-3 t / \tau} \\
& +\left(\operatorname{coth} x-6 / x+15 \operatorname{coth} x / x^{2}-15 / x^{3}\right)\left[\left(5 \beta_{\lambda \mu \nu} \hat{\mu}_{\lambda} \hat{\mu}_{\mu} \hat{\mu}_{\nu}-2 \beta_{\lambda \lambda \mu} \hat{\mu}_{\mu}-\beta_{\lambda \mu \mu} \hat{\mu}_{\lambda}\right)\right. \\
& \left.\times \cos \phi^{\prime} \sin \varsigma\left(1+5 \cos 2 \phi-2 \cos ^{2} \phi \cos 2 \varsigma-4 \sin 2 \phi \tan \phi^{\prime}\right)\right]\left.\mathrm{e}^{-6 t / \tau}\right|^{2} .
\end{aligned}
$$


Firestone et al. [7] measured SHG using a $Q$-switched Nd:YAG laser using p-p polarised geometry $\left(\phi=\phi^{\prime}=0\right)$ in which case Eq. (33) predicts for the harmonic intensity

$$
\begin{aligned}
\mathscr{J}_{\mathrm{pp}}= & \frac{\mathscr{B}^{(2)}(\Delta k)}{400} \mid 4(\operatorname{coth} x-1 / x) \sin \varsigma\left[\left(2 \beta_{\lambda \mu \mu} \hat{\mu}_{\lambda}-\beta_{\lambda \lambda \mu} \hat{\mu}_{\mu}\right)-\left(3 \beta_{\lambda \lambda \mu} \hat{\mu}_{\mu}-\beta_{\lambda \mu \mu} \hat{\mu}_{\lambda}\right) \cos 2 \varsigma\right] \mathrm{e}^{-t / \tau} \\
& +\left(\operatorname{coth} x-6 / x+15 \operatorname{coth} x / x^{2}-15 / x^{3}\right) \\
& \times\left.\left[\left(5 \beta_{\lambda \mu \nu} \hat{\mu}_{\lambda} \hat{\mu}_{\mu} \hat{\mu}_{\nu}-2 \beta_{\lambda \lambda \mu} \hat{\mu}_{\mu}-\beta_{\lambda \mu \mu} \hat{\mu}_{\lambda}\right) \sin \varsigma(3-\cos 2 \varsigma)\right] \mathrm{e}^{-6 t / \tau}\right|^{2}
\end{aligned}
$$

Instead of the intensity, the authors prefer to consider a second harmonic coefficient $d_{33}(t)$ defined by their own terms as

$$
\frac{d_{33}(t)}{d_{33}(0)}=\left(\frac{\mathscr{F}_{\mathrm{pp}}(t)}{\mathscr{F}_{\mathrm{pp}}(0)}\right)^{1 / 2},
$$

where $t=0$ is taken as the moment that the external field is cut and relaxation begins. Comparing this to Eq. (34) we would expect the data to relax as a bi-exponential function with components whose exponents are in the ratio of $1: 6$. The reported experimental relaxation times, for data fitted to a bi-exponential form, are $\tau_{1}=0.37$ $\min$ and $\tau_{2}=2.84 \mathrm{~min}$, giving a ratio of $1: 7.7$. This is in broad agreement with the theoretical value predicted here, considering that the second harmonic signal is stated as being reproducible to $\pm 10 \%$. Of course the similarity may be coincidental - more accurate data, perhaps at higher temperatures where a diffusional model may be more appropriate, are called for. The development of polarisation studies may also prove fruitful in such experiments.

\subsection{Second harmonic generation from a normal distribution of orientations}

The poled polymers discussed above may produce useful nonlinear optical devices but will always be restricted to low temperature applications. Recent experiments involving composites of organic molecules and molecular sieves suggest the possibility of producing nonlinear devices with a high thermal stability due to the mineral nature of the sieves [8]. Organic molecules trapped within sieves can become preferentially orientated by, for example, intermolecular hydrogen bonding; this is thought to be the case for p-nitroaniline (PNA) which forms very well aligned dipole chains within sieves. We shall consider the case of molecules aligned but with their dipole moments having a normal distribution of orientations about a mean orientation at an angle $\theta_{0}$ to the laboratory fixed direction. In other words the energetically most favoured orientations generate a conical locus of semi-angle $\theta_{0}$ about the axis $\hat{\boldsymbol{u}}$. Hence, the orientational distribution function is given by

$$
f(\theta)=\exp \left\{-\left[\left(\theta-\theta_{0}\right) / a\right]^{2}\right\}
$$

where the constant $a$ could be taken as temperature dependent if thermal effects need to be considered. Using Eq. (11), the second harmonic intensity in Eq. (27) becomes

$$
\mathscr{I}=\mathscr{B}^{(2)}(\Delta \boldsymbol{k})\left|\sum_{n=1}^{3} A_{n} B_{n}(\hat{\boldsymbol{u}}, \hat{\boldsymbol{\mu}})\right|^{2},
$$

where the coefficients $A_{n}$ are given by

$$
\begin{aligned}
& A_{1}=\frac{3}{2}\left(\int_{0}^{\pi} \sin 2 \theta \exp \left\{-\left[\left(\theta-\theta_{0}\right) / a\right]^{2}\right\} \mathrm{d} \theta / \int_{0}^{\pi} \sin \theta \exp \left\{-\left[\left(\theta-\theta_{0}\right) / a\right]^{2}\right\} \mathrm{d} \theta\right), \\
& A_{2}=\frac{15}{8}\left(\int_{0}^{\pi} \sin 3 \theta \exp \left\{-\left[\left(\theta-\theta_{0}\right) / a\right]^{2}\right\} \mathrm{d} \theta / \int_{0}^{\pi} \sin \theta \exp \left\{-\left[\left(\theta-\theta_{0}\right) / a\right]^{2}\right\} \mathrm{d} \theta\right)-\frac{5}{8},
\end{aligned}
$$




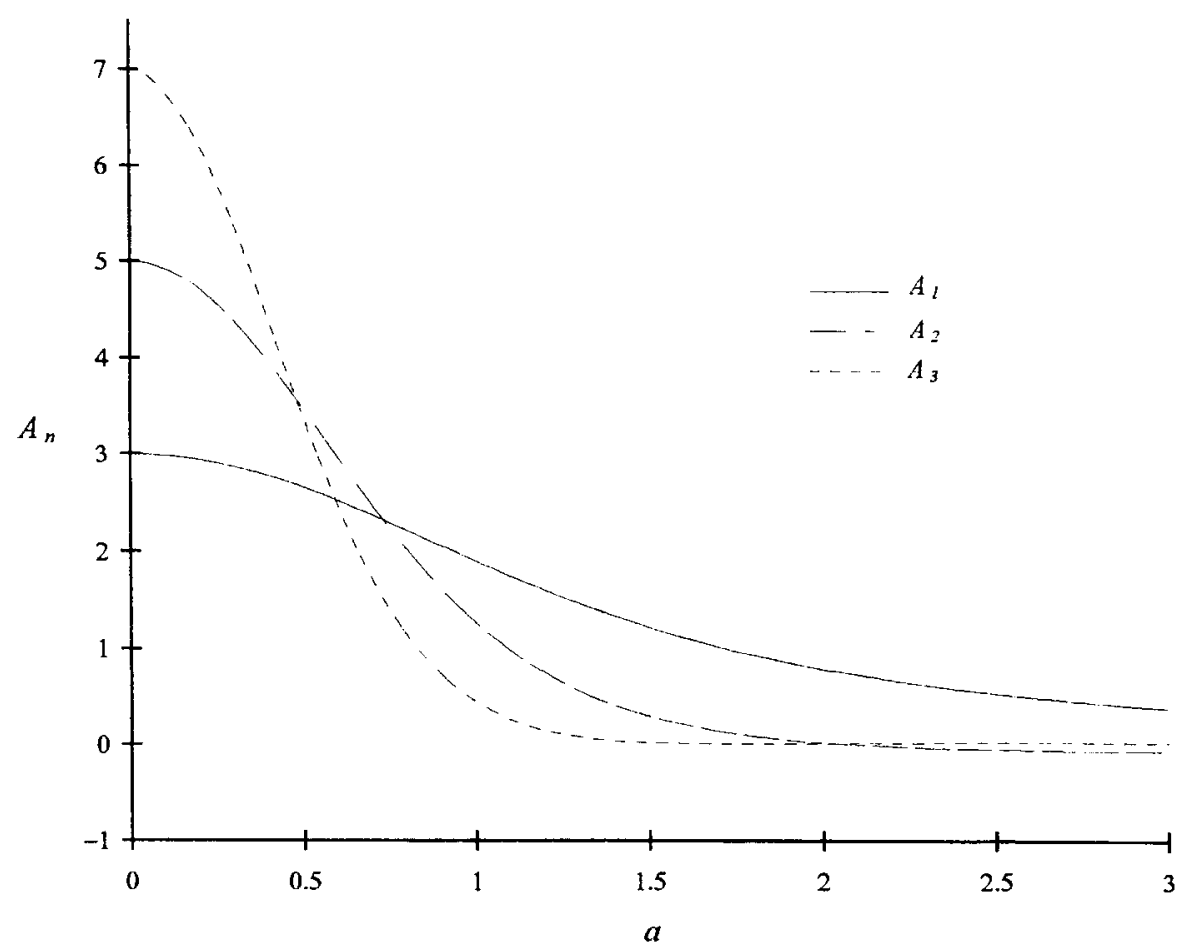

Fig. 3. Graph showing the effect of tightening up the normal distribution of orientations on the coefficients $A_{n}$ of Eqs. (38)-(40). The value of $\theta_{0}$ has been taken as zero and smaller $a$ values correspond to a more tightly aligned set of molecules.

$$
A_{3}=\frac{35}{16}\left(\int_{0}^{\pi} \sin 4 \theta \exp \left\{-\left[\left(\theta-\theta_{0}\right) / a\right]^{2}\right\} \mathrm{d} \theta / \int_{0}^{\pi} \sin \theta \exp \left\{-\left[\left(\theta-\theta_{0}\right) / a\right]^{2}\right\} \mathrm{d} \theta\right)-\frac{7}{12} A_{1}
$$

These coefficients clearly depend on the "tightness" of the alignment, as dictated by $a$, and on the mean preferred direction $\theta_{0}$. A graphical illustration of their variation with $a$ is shown in Fig. 3 for the case $\theta_{0}=0$. We see that in the limit as $a$ becomes large the coefficients all tend to zero, i.e. no SHG occurs when the molecules are not preferentially aligned. As $a$ tends to zero the molecules become perfectly aligned and the limits

$$
\left.\begin{array}{l}
\lim _{a \rightarrow 0} A_{1}=3 \cos \theta_{0}=3 P_{1}\left(\cos \theta_{0}\right) \\
\lim _{a \rightarrow 0} A_{2}=\frac{5}{2}\left(3 \cos ^{2} \theta_{0}-1\right)=5 P_{2}\left(\cos \theta_{0}\right) \\
\lim _{a \rightarrow 0} A_{3}=\frac{7}{2} \cos \theta_{0}\left(5 \cos ^{2} \theta_{0}-3\right)=7 P_{3}\left(\cos \theta_{0}\right)
\end{array}\right\}
$$

are obtained. In the perfectly aligned case, with $\theta_{0}=0$, we therefore have

$$
\mathscr{f}=\mathscr{B}^{(2)}(\Delta \boldsymbol{k})\left|3 B_{1}(\hat{\boldsymbol{\mu}}, \hat{\boldsymbol{\mu}})+5 B_{2}(\hat{\boldsymbol{u}}, \hat{\boldsymbol{\mu}})+7 B_{3}(\hat{\boldsymbol{u}}, \hat{\boldsymbol{\mu}})\right|^{2} .
$$

In passing, we can also note that results for a completely isotropic distribution of orientations can be recovered by averaging over $\theta_{0}$ in the limits of Eq. (41), giving $A_{1}=A_{2}=A_{3}=0$.

We can now use Eq. (37) to predict the polarisation characteristics of the second harmonic produced by molecules whose orientations are normally distributed, comparing our predictions with studies that have been 


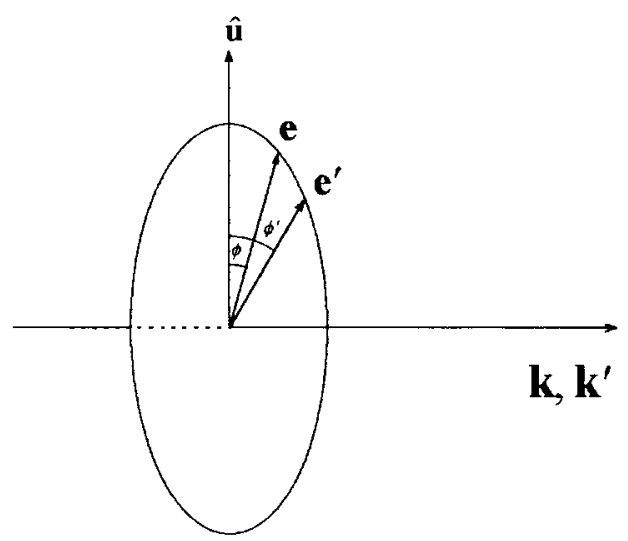

Fig. 4. Diagram to show the geometry used in experiments measuring the SHG from molecules trapped in a molecular sieve. Both $\phi$ and $\phi^{\prime}$ are positive in the sense of an anti-clockwise rotation from the direction of preferred molecular orientation $\hat{\boldsymbol{u}}$ about the positive $\boldsymbol{k}$ direction.

performed on molecules contained within sieves. We shall adopt the beam configuration shown in Fig. 4, setting $\theta_{0}=0$ from now on to coincide with the experimental arrangement used by these authors [8]. The second harmonic signal is measured in the forward direction and we take the plane polarised beams to be such that the fundamental makes an angle $\phi$ to the orientation direction (the molecular sieve axis), the second harmonic making an angle $\phi^{\prime}$ with respect to the same axis. Eq. (37) now yields

$$
\begin{aligned}
\mathscr{I}= & \mathscr{B}^{(2)}(\Delta k) \mid \frac{A_{1}}{15}\left[\left(2 \beta_{\lambda \mu \mu} \hat{\mu}_{\lambda}-\beta_{\lambda \lambda \mu} \hat{\mu}_{\mu}\right) \cos \phi^{\prime}+\left(3 \beta_{\lambda \lambda \mu} \hat{\mu}_{\mu}-\beta_{\lambda \mu \mu} \hat{\mu}_{\lambda}\right) \cos \phi \cos \left(\phi-\phi^{\prime}\right)\right] \\
& +\left.\frac{A_{3}}{70}\left(5 \beta_{\lambda \mu \nu} \hat{\mu}_{\lambda} \hat{\mu}_{\mu} \hat{\mu}_{\nu}-2 \beta_{\lambda \lambda \mu} \hat{\mu}_{\mu}-\beta_{\lambda \mu \mu} \hat{\mu}_{\lambda}\right)\left[5 \cos ^{2} \phi \cos \phi^{\prime}-2 \cos \phi \cos \left(\phi-\phi^{\prime}\right)-\cos \phi^{\prime}\right]\right|^{2},
\end{aligned}
$$

where $A_{1}$ and $A_{3}$ are given by Eqs. (38) and (40) respectively. This expression, (43), allows us to calculate the second harmonic intensity for any combination of angles $\phi$ and $\phi^{\prime}$. For comparison to the work of Marlow et al. we shall define $\mathscr{I}_{\|}(\phi)$ as the intensity of the second harmonic light polarised parallel to the orientation direction $\hat{\boldsymbol{u}}$ when the incident laser polarisation is at an angle $\phi, \mathscr{J}_{\perp}(\phi)$ as the second harmonic intensity perpendicular to $\hat{\boldsymbol{u}}$ for the same input radiation and $\mathscr{F}_{\phi^{\prime}}(\pi / 4)$ as the SHG at an angle $\phi^{\prime}$ when the incident light is set at an angle of $\pi / 4$ to $\hat{\boldsymbol{u}}$. Our expressions for these quantities are easily found from Eq. (43):

$$
\begin{aligned}
\mathscr{I}_{\|}(\phi)= & \mathscr{B}^{(2)}(\Delta k) \mid \frac{A_{1}}{15}\left[\left(2 \beta_{\lambda \mu \mu} \hat{\mu}_{\lambda}-\beta_{\lambda \lambda \mu} \hat{\mu}_{\mu}\right)+\left(3 \beta_{\lambda \lambda \mu} \hat{\mu}_{\mu}-\beta_{\lambda \mu \mu} \hat{\mu}_{\lambda}\right) \cos ^{2} \phi\right] \\
& +\left.\frac{A_{3}}{70}\left(5 \beta_{\lambda \mu \nu} \hat{\mu}_{\lambda} \hat{\mu}_{\mu} \hat{\mu}_{\nu}-2 \beta_{\lambda \lambda \mu} \hat{\mu}_{\mu}-\beta_{\lambda \mu \mu} \hat{\mu}_{\lambda}\right)\left(3 \cos ^{2} \phi-1\right)\right|^{2} \\
\mathscr{J}_{\perp}(\phi)= & \mathscr{F}^{(2)}(\Delta k) \sin ^{2} \phi \cos ^{2} \phi \mid \frac{A_{1}}{15}\left(3 \beta_{\lambda \lambda \mu} \hat{\mu}_{\mu}-\beta_{\lambda \mu \mu} \hat{\mu}_{\lambda}\right) \\
& -\left.\frac{A_{3}}{35}\left(5 \beta_{\lambda \mu \nu} \hat{\mu}_{\lambda} \hat{\mu}_{\mu} \hat{\mu}_{\nu}-2 \beta_{\lambda \lambda \mu} \hat{\mu}_{\mu}-\beta_{\lambda \mu \mu} \hat{\mu}_{\lambda}\right)\right|^{2}
\end{aligned}
$$




$$
\begin{aligned}
\mathscr{I}_{\phi^{\prime}}\left(\frac{\pi}{4}\right)= & \mathscr{B}^{(2)}(\Delta k) \mid\left(\frac{A_{1}}{30}\left(3 \beta_{\lambda \mu \mu} \hat{\mu}_{\lambda}+\beta_{\lambda \lambda \mu} \hat{\mu}_{\mu}\right)+\frac{A_{3}}{140}\left(5 \beta_{\lambda \mu \nu} \hat{\mu}_{\lambda} \hat{\mu}_{\mu} \hat{\mu}_{\nu}-2 \beta_{\lambda \lambda \mu} \hat{\mu}_{\mu}-\beta_{\lambda \mu \mu} \hat{\mu}_{\lambda}\right)\right) \cos \phi^{\prime} \\
& +\left.\left(\frac{A_{1}}{30}\left(3 \beta_{\lambda \lambda \mu} \hat{\mu}_{\mu}-\beta_{\lambda \mu \mu} \hat{\mu}_{\lambda}\right)-\frac{A_{3}}{70}\left(5 \beta_{\lambda \mu \nu} \hat{\mu}_{\lambda} \hat{\mu}_{\mu} \hat{\mu}_{\nu}-2 \beta_{\lambda \lambda \mu} \hat{\mu}_{\mu}-\beta_{\lambda \mu \mu} \hat{\mu}_{\lambda}\right)\right) \sin \phi^{\prime}\right|^{2} .
\end{aligned}
$$

Marlow et al. [8] have carried out experiments using a frequency doubled Nd: YAG laser and (dimethylamino)benzonitrile (DMABN) trapped in $\mathrm{AlPO}_{4}-5$ molecular sieves, empirically fitting their data to expressions of the form

$$
\begin{aligned}
\mathscr{I}_{\|}(\phi) & =a^{2} \cos ^{4} \phi+b^{2} \sin ^{4} \phi \equiv\left(a^{2}+b^{2}\right) \cos ^{4} \phi-2 b^{2} \cos ^{2} \phi+b^{2}, \\
\mathscr{I}_{\perp}(\phi) & =c(\cos \phi \sin \phi)^{2} \equiv c \sin ^{2} \phi \cos ^{2} \phi, \\
\mathscr{F}_{\phi^{\prime}}\left(\frac{\pi}{4}\right) & =A^{2} \sin ^{2}\left(\phi^{\prime}+\gamma\right)+B^{2} \sin ^{2} \phi^{\prime} \\
& \equiv A^{2} \sin ^{2} \gamma \cos ^{2} \phi^{\prime}+A^{2} \sin 2 \gamma \cos \phi^{\prime} \sin \phi^{\prime}+\left(A^{2} \cos ^{2} \gamma+B^{2}\right) \sin ^{2} \phi^{\prime},
\end{aligned}
$$

where their parameters $a, b, c, A, B$ and $\gamma$ necessarily have real values to be determined. We see that the form predicted by Eq. (45) for $\mathscr{I}_{\perp}$ tallies directly with the expression (48) used by Marlow et al., and fits their data well. The expressions for $\mathscr{I}_{\|}$and $\mathscr{F}_{\phi^{\prime}}(\pi / 4)$, do not so obviously tally unless certain relationships hold amongst the hyperpolarisability components. Fig. 5 shows a comparison of the polarisation behaviour given by Marlow et al. with that predicted here, on the assumption that all hyperpolarisability components are real. We note that the data obtained by Marlow et al. fits their expressions reasonably well, but its accuracy is unlikely to offer a basis for differentiating the two suggested forms for $\mathscr{I}_{\|}$. More obviously there is a large discrepancy between the experimental data for $\mathcal{F}_{\phi^{\prime}}(\pi / 4)$ and our proposed form given by Eq. (46), the experimental data of Ref. [8] clearly not going to zero for particular values of $\phi^{\prime}$. The resolution of this problem proves to shed new light on the nature of the nonlinear optical response at the molecular level, as we shall now demonstrate.

One possible cause for the differences apparent in Fig. 5 is that our results were there based on the assumption that all components of the molecular hyperpolarisability were real. Such a supposition would only be valid if there were no resonance behaviour coming into play. It is interesting that of the three Eqs. (44)-(46), only (45) has the same polarisation dependence irrespective of the complex or real nature of the hyperpolarisability components therein. Before pursuing the possible effects of resonant behaviour further it is worth considering whether this might be a possibility for the experiments of Marlow et al. For DMABN excited states are reported at 32100 and $34780 \mathrm{~cm}^{-1}$; as the latter has an approximate full width at half maximum [12] of $4000 \mathrm{~cm}^{-1}$, it substantially overlaps the second harmonic frequency of $37594 \mathrm{~cm}^{-1}$. Indeed these absorption frequencies should be treated with some caution because they relate to DMABN dissolved in a $1: 1$ mixture of 2-methylbutane and 2,2,4-trimethylpentane, rather than subjected to the conditions used in the SHG experiments. Bearing these points in mind, it appears a realistic proposition that the hyperpolarisabilities may have some resonant character and thereby acquire complex character. To accommodate this possibility we now note that the polarisation characteristics of Eqs. (44) and (46) can in general be expressed in the form

$$
\mathscr{J}_{\|}(\phi)=\left|c_{1}+c_{2} \mathrm{e}^{\mathrm{i} \delta} \cos ^{2} \phi\right|^{2}
$$

and

$$
\mathscr{I}_{\phi^{\prime}}\left(\frac{\pi}{4}\right)=\left|c_{3} \cos \phi^{\prime}+c_{4} \mathrm{e}^{\mathrm{i} \delta^{\prime}} \sin \phi^{\prime}\right|^{2}
$$

where the coefficients $c_{i}$ and relative phase factors $\delta$ and $\delta^{\prime}$ are real. If the hyperpolarisability components were themselves real, these phase factors would vanish, non-zero values being permitted if the hyperpolarisability components are complex. In this sense, Fig. 5 represents the best fit for $\delta=\delta^{\prime}=0$. Fig. 6 shows the effect that complex hyperpolarisability can have on the polarisation characteristics of $\mathscr{F}_{\|}$and $\mathscr{I}_{\phi^{\prime}}(\pi / 4)$. Clearly the 

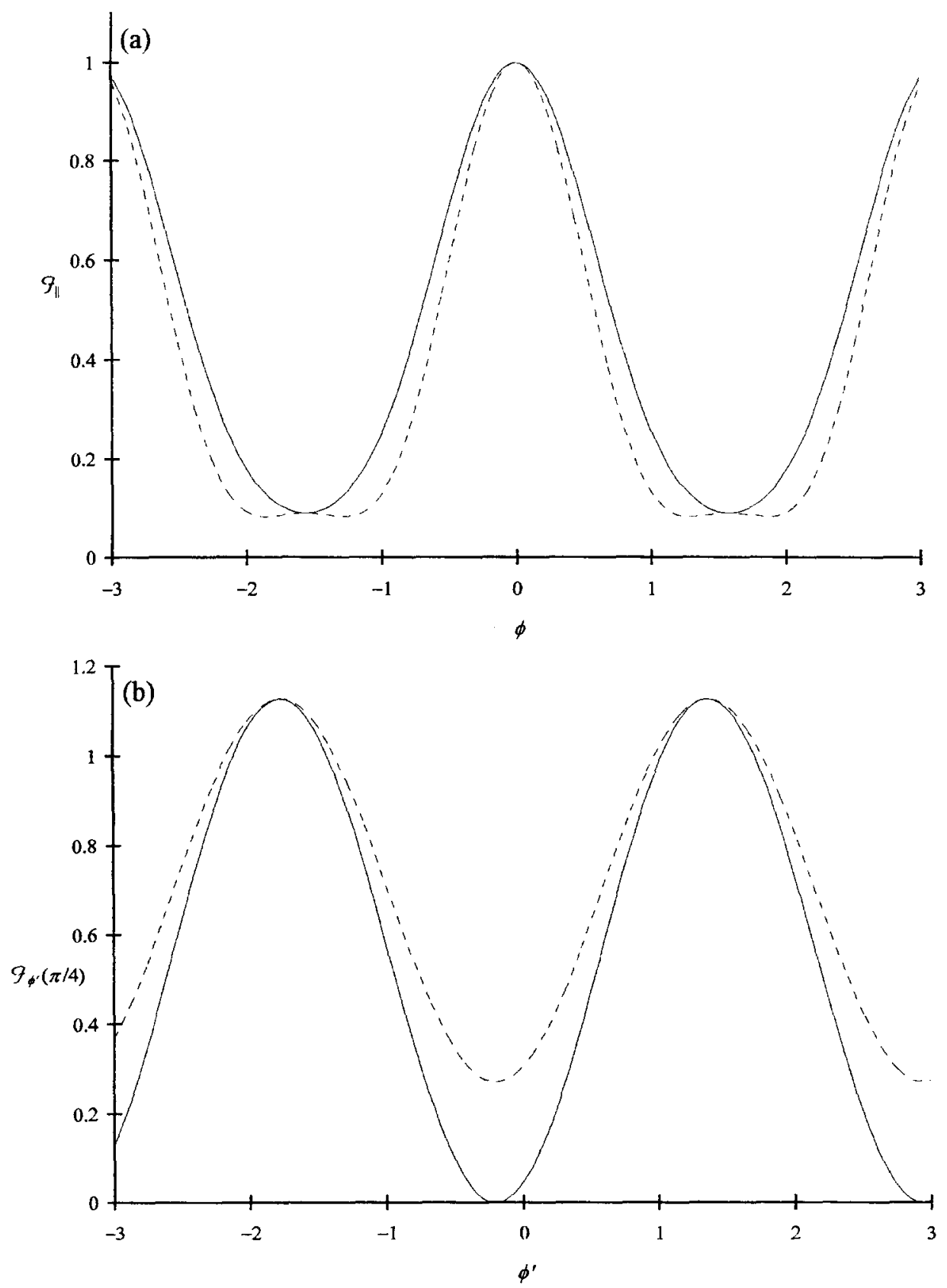

Fig. 5. A graphical comparison of the polarisation characteristics predicted by Eq. (44) and (46) to those based on the expressions of Marlow et al., Ref. [8]. In both cases a solid line represents out model and the dashed line that of Marlow et al. The graphs show (a) $\mathscr{F}_{\|}$, arranged so that both traces are normalised to unity and agree at $\phi=\pi / 2$, and (b) $\mathscr{I}_{\phi^{\prime}}(\pi / 4)$, arranged so that both share common maxima at the experimentally observed values of $\phi^{\prime}$.

inclusion of near-resonance behaviour can properly account for the experimentally obtained polarisation characteristics. One corollary is that we would expect polarisation studies to provide the means for separating out the real and imaginary parts of the hyperpolarisability. 

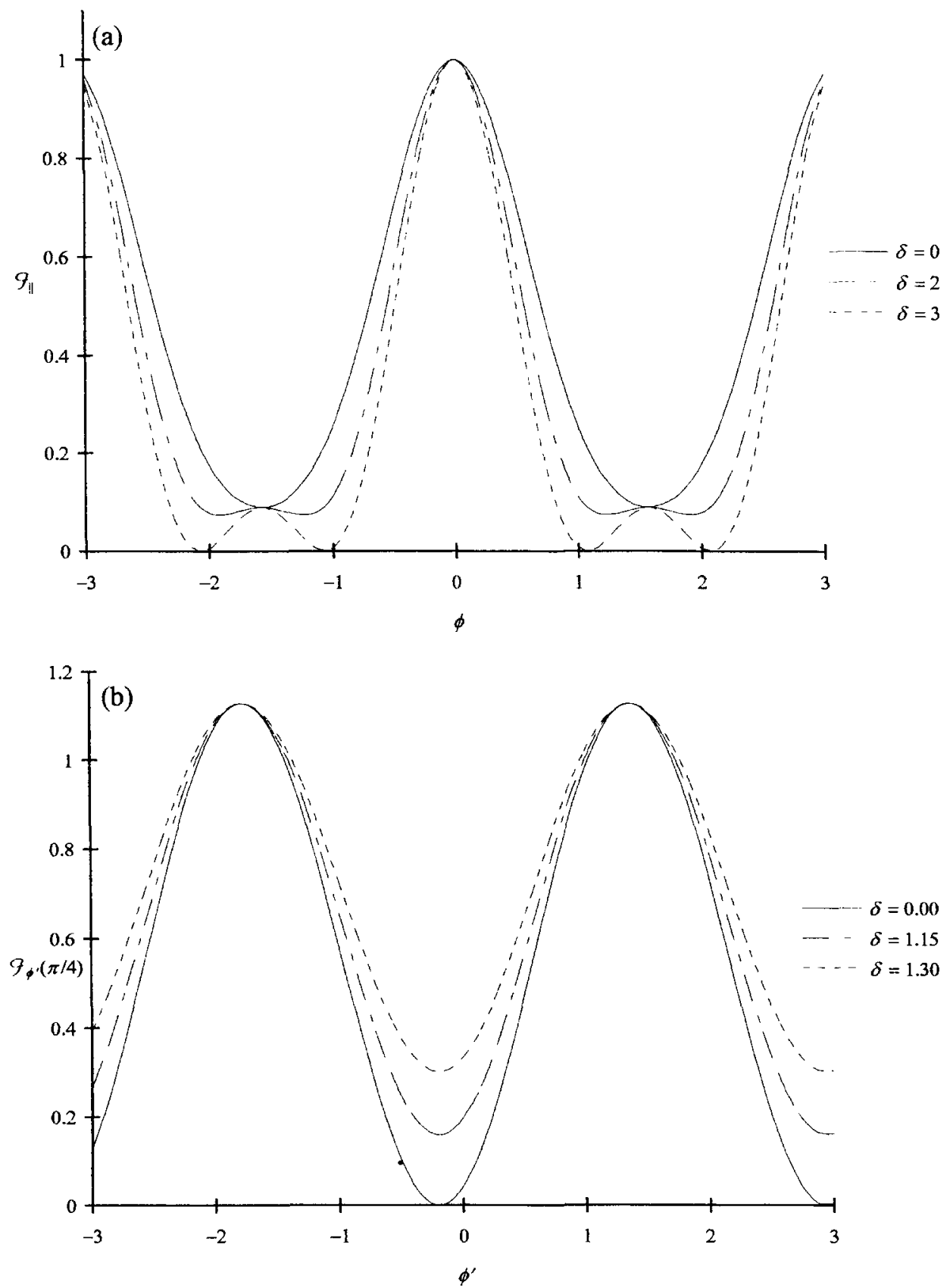

Fig. 6. The change of the polarisation characteristics of the quantities $\mathscr{I}_{\|}$and $\mathscr{F}_{\phi^{\prime}}(\pi / 4)$ on allowing for complex hyperpolarisabilities. The graphs show (a) $\mathscr{F}_{\|}$, arranged so that all traces are normalised to unity and agree at $\phi=\pi / 2$, and (b) $\mathscr{I}_{\phi^{\prime}}(\pi / 4)$, arranged so that all share common maxima at the $\phi^{\prime}$ values observed experimentally by Marlow et al., Ref. [8]. The phase factors $\delta$ and $\delta^{\prime}$ are those appearing in Eqs. (50) and (51), zero values corresponding to the predictions obtained for real hyperpolarisabilities. 


\subsection{Second harmonic generation from molecules on surfaces}

To conclude, we consider application of our theory to surface SHG. Since a coherent second harmonic signal cannot be generated within the bulk of an isotropic or centrosymmetric medium, the observation of such signals by surface reflection is a technique widely employed for the characterisation of molecules at, or adsorbed on, surfaces. To develop the theory we consider again the configuration shown in Fig. 2, taking the reference direction $\hat{\boldsymbol{u}}$ to be normal to the surface, $\varsigma$ as the angle of incidence and with $\phi, \phi^{\prime}$ as shown. The full expression for the second harmonic intensity is again given by Eq. (27), which, assuming a normal distribution of molecular orientations, now takes the form

$$
\begin{aligned}
\mathscr{J}= & \mathscr{B}^{(2)}(\Delta k) \mid \frac{A_{1}}{15} \sin \varsigma\left[\left(2 \beta_{\lambda \mu \mu} \hat{w}_{\lambda}-\beta_{\lambda \lambda \mu} \hat{w}_{\mu}\right) \cos \phi^{\prime}\right. \\
& \left.+\left(3 \beta_{\lambda \lambda \mu} \hat{w}_{\mu}-\beta_{\lambda \mu \mu} \hat{w}_{\lambda}\right) \cos \phi\left(\sin \phi \sin \phi^{\prime}-\cos \phi \cos \phi^{\prime} \cos 2 \varsigma\right)\right] \\
& -\frac{A_{2}}{10} \epsilon_{\lambda \mu \tau} \beta_{\lambda \mu \nu} \hat{w}_{\nu} \hat{w}_{\tau} \cos \phi \sin \left(\phi+\phi^{\prime}\right) \sin 2 \varsigma \\
& +\frac{A_{3}}{280}\left(5 \beta_{\lambda \mu \nu} \hat{w}_{\lambda} \hat{w}_{\mu} \hat{w}_{\nu}-2 \beta_{\lambda \lambda \mu} \hat{w}_{\mu}-\beta_{\lambda \mu \mu} \hat{w}_{\lambda}\right) \cos \phi^{\prime} \\
& \times\left.\sin \varsigma\left(1+5 \cos 2 \phi-2 \cos ^{2} \phi \cos 2 \varsigma-4 \sin 2 \phi \tan \phi^{\prime}\right)\right|^{2} .
\end{aligned}
$$

The coefficients $A_{i}\left(a, \theta_{0}\right)$ are, in general, given by Eq. (38) to (40), with $a$ representing the extent of orientational distribution and $\theta_{0}$ the mean orientation of the molecules on the surface. If polarisation studies can elucidate the values of these coefficients $A_{i}$ then it may be possible to deduce a value for $\theta_{0}$ provided we have knowledge of the orientational distribution factor $a$. This may be possible by expressing the temperature dependence of the distribution through the factor $a$. Hence we might expect that coupled polarisation and temperature studies are necessary to gain the fullest information about molecular orientation at surfaces.

Eq. (52) is quite general. For its typical application, we now consider SHG experiments recently performed by Barnoski et al. [3] using monolayers of vitamin $\mathrm{K}_{1}$ on the surface of an aqueous medium, these studies being conducted with the intention of determining the orientation of the vitamin on the surface. These workers collected data for the case when $\varsigma=\pi / 4$, the second harmonic signal having polarisations of either $\phi^{\prime}=0$ (p-polarisation) or $\phi^{\prime}=\pi / 2$ (s-polarisation). Denoting the signal in the former case as $\mathcal{F}_{\mathrm{p}}$ and in the latter as $\mathscr{F}_{\mathrm{s}}$ we have from Eq. (52)

$$
\begin{aligned}
\mathscr{F}_{\mathrm{p}}= & \mathscr{B}^{(2)}(\Delta k) \mid \frac{A_{1}}{15 \sqrt{2}}\left(2 \beta_{\lambda \mu \mu} \hat{w}_{\lambda}-\beta_{\lambda \lambda \mu} \hat{w}_{\mu}\right)-\frac{A_{2}}{20} \epsilon_{\lambda \mu \tau} \beta_{\lambda \mu \nu} \hat{w}_{\nu} \hat{w}_{\tau} \sin 2 \phi \\
& +\left.\frac{A_{3}}{280 \sqrt{2}}\left(5 \beta_{\lambda \mu \nu} \hat{w}_{\lambda} \hat{w}_{\mu} \hat{w}_{\nu}-2 \beta_{\lambda \lambda \mu} \hat{w}_{\lambda}-\beta_{\lambda \mu \mu} \hat{w}_{\lambda}\right)(1+5 \cos 2 \phi)\right|^{2},
\end{aligned}
$$

and

$$
\begin{aligned}
\mathscr{J}_{\mathrm{s}}= & \mathscr{\beta}^{(2)}(\Delta \boldsymbol{k}) \mid\left(\frac{A_{1}}{30 \sqrt{2}}\left(3 \beta_{\lambda \lambda \mu} \hat{w}_{\mu}-\beta_{\lambda \mu \mu} \hat{w}_{\lambda}\right)-\frac{A_{3}}{70 \sqrt{2}}\left(5 \beta_{\lambda \mu \nu} \hat{w}_{\lambda} \hat{w}_{\mu} \hat{w}_{\nu}-2 \beta_{\lambda \lambda \mu} \hat{w}_{\mu}-\beta_{\lambda \mu \mu} \hat{w}_{\lambda}\right)\right) \sin 2 \phi \\
& -\left.\frac{A_{2}}{20} \epsilon_{\lambda \mu \tau} \beta_{\lambda \mu \nu} \hat{w}_{\nu} \hat{w}_{\tau}(1+\cos 2 \phi)\right|^{2} .
\end{aligned}
$$

For the expanded monolayer Barnoski et al. observed both $\mathscr{I}_{\mathrm{p}}$ and $\mathscr{F}_{\mathrm{s}}$ to be zero, indicating that the molecules are not preferentially aligned (as noted earlier, this is consistent with having all $A_{i}=0$.) On compression, 
however, it was found that steric interaction between molecules engenders SHG with p-polarisation, though not with s-polarisation. In the light of Eqs. (53) and (54) this behaviour is at first sight somewhat puzzling. It suggests that in this particular case we must have the following identities:

$$
\begin{aligned}
& A_{2} \epsilon_{\lambda \mu \tau} \beta_{\lambda \mu \nu} \hat{w}_{\nu} \hat{w}_{\tau} \equiv 0, \\
& \left(\frac{A_{1}}{3}\left(3 \beta_{\lambda \lambda \mu} \hat{w}_{\mu}-\beta_{\lambda \mu \mu} \hat{w}_{\lambda}\right)-\frac{A_{3}}{7}\left(5 \beta_{\lambda \mu \nu} \hat{w}_{\lambda} \hat{w}_{\mu} \hat{w}_{\nu}-2 \beta_{\lambda \lambda \mu} \hat{w}_{\mu}-\beta_{\lambda \mu \mu} \hat{w}_{\lambda}\right)\right) \equiv 0,
\end{aligned}
$$

without the trivial $A_{1}=A_{2}=A_{3}=0$ solution being admissible. Relations (55) and (56) in turn imply that (53) can be written in the form

$$
\mathscr{F}_{\mathrm{p}}=\frac{\mathscr{B}^{(2)}(\Delta k) A_{1}^{2}}{1152}\left|\left(3 \beta_{\lambda \mu \mu} \hat{w}_{\lambda}-\beta_{\lambda \lambda \mu} \hat{w}_{\mu}\right)+\left(3 \beta_{\lambda \lambda \mu} \hat{w}_{\mu}-\beta_{\lambda \mu \mu} \hat{w}_{\lambda}\right) \cos 2 \phi\right|^{2} .
$$

This form does indeed match that shown by the experimental data obtained by Barnoski et al.; the observed SHG is symmetrical about $\phi=\pi / 2$ where the signal takes on a minimum value, and the maximum signal occurs at $\phi=0$ provided

$$
\left|\beta_{\lambda \lambda \mu} \hat{w}_{\mu}+\beta_{\lambda \mu \mu} \hat{w}_{\lambda}\right|^{2}>4\left|\beta_{\lambda \lambda \mu} \hat{w}_{\mu}-\beta_{\lambda \mu \mu} \hat{w}_{\lambda}\right|^{2} .
$$

Fitting experimental data to Eq. (57) will now yield information pertaining to the hyperpolarisability tensor of the molecule and not its orientation.

At this juncture it is worth commenting on a commonly employed assumption concerning long, rod-like molecules like vitamin $\mathrm{K}_{1}$. It is often approximated that such molecules should possess a single, dominating component in their hyperpolarisability tensor. If we were to assume this to be the case here, as indeed Barnoski et al. have in their analysis, denoting this component by $\beta_{z z z}$ and taking the molecule fixed vector $\hat{\boldsymbol{w}}$ to be along this direction so that $\hat{w}_{\alpha}=\delta_{\alpha z}$, Eq. (57) would give

$$
\mathscr{J}_{\mathrm{p}}=\frac{\mathscr{B}^{(2)}(\Delta \boldsymbol{k}) A_{1}^{2}\left|\beta_{z z z}\right|^{2}}{288}|1+\cos 2 \phi|^{2} .
$$

This would require that the minimum value of the p-polarised signal should be zero, whereas it is clear from the data that this is not so. A less drastic assumption might be a consideration that there are three non-zero and independent components of the hyperpolarisability tensor, as is consistent with a molecule of $\mathrm{C}_{\infty \mathrm{v}}$ symmetry [13], namely;

$$
\begin{aligned}
& \beta_{z z z}=\beta_{\|}, \\
& \beta_{z x x}=\beta_{z y y}=\beta_{\perp}, \\
& \beta_{x z x}=\beta_{x x z}=\beta_{y z y}=\beta_{y y z}=\beta_{\perp}+\Delta \beta_{\perp} .
\end{aligned}
$$

Using these definitions the expressions for the p- and s-polarised second harmonic given in Eqs. (53) and (54) become respectively

$$
\begin{aligned}
& \mathscr{I}_{\mathrm{p}}=\frac{\mathscr{B}^{(2)}(\Delta k)}{50}\left|\frac{A_{1}}{3}\left(\beta_{\|}+2 \beta_{\perp}-2 \Delta \beta_{\perp}\right)+\frac{A_{3}}{28}\left(\beta_{\|}-3 \beta_{\perp}-2 \Delta \beta_{\perp}\right)(1+5 \cos 2 \phi)\right|^{2}, \\
& \mathscr{J}_{\mathrm{s}}=\frac{\mathscr{B}^{(2)}(\Delta k)}{50}\left|\frac{A_{1}}{3}\left(\beta_{\|}+2 \beta_{\perp}+3 \Delta \beta_{\perp}\right)-\frac{A_{3}}{7}\left(\beta_{\|}-3 \beta_{\perp}-2 \Delta \beta_{\perp}\right)\right|^{2} \sin ^{2} 2 \phi .
\end{aligned}
$$

This still indicates that if we were to treat vitamin $\mathrm{K}_{\text {, }}$ as a rod-like molecule possessing $\mathrm{C}_{x_{\mathrm{v}}}$ symmetry we would nonetheless expect there to be an s-polarised second harmonic signal under appropriate input polarisation 
conditions. For p- or s-polarised input ( $\phi=0$ or $\pi / 2$ respectively) the s-polarised harmonic here vanishes, as is commonly observed.

\section{Conclusion}

We have presented here a method of obtaining orientational averages of direction dependent molecular properties weighted by Legendre polynomial functions, showing by specific reference to second harmonic generation how the method may be applied. The examples that we have considered illustrate the diversity of information of both practical and theoretical interest that may be derived from application of the theory, which is readily adaptable to other experimental arrangements. Our results show that polarisation studies of SHG, ideally coupled with temporal and/or temperature dependence studies, should be capable of yielding further insight into phenomena related to molecular orientation and the mechanisms underlying the molecular response. Finally, it is shown how great caution should be exercised in the application of over-simplistic models in the studies of SHG from surfaces.

\section{Acknowledgements}

We thank Dr. S.R. Meech for helpful comments on the manuscript, and we are pleased to acknowledge that the work is funded by the Engineering and Physical Sciences Research Council.

\section{References}

[1] D.L. Andrews, Modem nonlinear optics, Part 2, eds. M. Evans and S. Kielich; Advan. Chem. Phys. 85 (1993) 545.

[2] R.M. Corn and D.A. Higgens, Chem. Rev. 94 (1994) 107.

[3] A.A. Barnoski, G.S. Frysinger, G.L. Gaines, Jr. and G.M. Korenowski, Colloids Surfaces A 88 (1994) 123.

[4] D.L. Andrews, P. Allcock and A.A. Demidov, Chem. Phys. 190 (1995) 1.

[5] P. Allcock, D.L. Andrews, S.R. Meech and A.J. Wigman, Phys. Rev. A, in press.

[6] M. Eich, A. Sen, H. Looser, G.C. Bjorklund, J.D. Swalen, R. Twieg and D.Y. Yoon, J. Appl. Phys. 66 (1989) 2559.

[7] M.A. Firestone, M.A. Ratner, T.J. Marks, W. Lin and G.K. Wong, Macromolecules 28 (1995) 2260.

[8] F. Marlow, J. Caro, L. Werner, J. Kornatowski and S. Dähne, J. Phys. Chem. 97 (1993) 11286.

[9] D.L. Andrews and M.J. Harlow, Phys. Rev. A 29 (1984) 2796.

[10] P. Debye, Polar Molecules (Chemical Catalog Co., 1929).

[11] D.M. Burland, R.D. Miller and C.A. Walsh, Chem. Phys. 94 (1994) 31.

[12] G. Wermuth, Z. Naturforsch. A 38 (1983) 368.

[13] A.D. Buckingham, in: Nonlinear behaviour of molecules, atoms and ions in electric, magnetic or electromagnetic fields, ed. L. Néel (Elsevier, Amsterdam, 1979) p. 217.

[14] D.L. Andrews and B.S. Sherborne, J. Phys. B 19 (1986) 4265. 